\title{
Lipid metabolism in mycobacteria - insights using mass spectrometry-based lipidomics
}

Peter J. Crick ${ }^{1,2}$ and Xue Li Guan ${ }^{1,2,3, *}$

${ }^{1}$ Swiss Tropical and Public Health Institute, CH-4051 Basel, Switzerland

${ }^{2}$ University of Basel, CH-4000 Basel, Switzerland

${ }^{3}$ Lee Kong Chian School of Medicine, Nanyang Technological University, Singapore

*Corresponding author: xueli.guan@ ntu.edu.sg (X. L. Guan)

\begin{abstract}
Diseases including tuberculosis and leprosy are caused by species of the Mycobacterium genus and are a huge burden on global health, aggravated by the emergence of drug resistant strains. Mycobacteria have a high lipid content and complex lipid profile including several unique classes of lipid. Recent years have seen a growth in research focused on lipid structures, metabolism and biological functions driven by advances in mass spectrometry techniques and instrumentation, particularly the use of electrospray ionization. Here we review the contributions of lipidomics towards the advancement of our knowledge of lipid metabolism in mycobacterial species.
\end{abstract}

Keywords: Mycobacterium, Tuberculosis, Systems Biology, Mass Spectrometry, Lipidomics, Metabolism

Abbreviations: ABC, ATP binding cassette; BCG, Bacillus Calmette Guérin; ChIP-Seq, chromatin immunoprecipitation-sequencing; CoA, coenzyme A; DAG, diacylglycerol; DAT, diacyl trehalose; DDM, dideoxymycobactin; ESI, electrospray ionisation; FT-ICR, Fourier transform ion cyclotron resonance; GC, gas chromatography; HIV, human immunodeficiency virus; HPLC, high performance liquid chromatography; HRAM, high resolution-accurate mass; LC, liquid chromatography; LIT, linear ion trap; MDR, multidrug-resistant; MF, molecular feature; mmDAG, monomeromycolyl diacylglycerol; MPD, mannosyl $\beta$-1-phosphodolichol; MPI, mannosyl $\beta$-1-phosphoisoprenoid; MRM, 
multiple reaction monitoring; MS, mass spectrometry; MscL, mechano-sensitive channel of large conductance; $\mathrm{MS}^{\mathrm{n}}$, mass spectrometry with multistage fragmentation; NMR, nuclear magnetic resonance; NTM, non-tuberculosis mycobacteria; PAT, pentaacyl trehalose; PDIM, phthiocerol dimycocerosate; PGL, phenolic glycolipid; PIM, phosphatidyl-myo-inositol mannoside; SGL, sulfated glycolipid; SL, sulfolipid; SPE, solid phase extraction; TAG, triacylglycerol; TAT, triacyl trehalose; TB, tuberculosis; TDM, trehalose dimycolate; THL, tetrahydrolipistatin; TMM, trehalose monomycolate; UV, ultraviolet; XDR, extensively drug-resistant.

\section{Introduction}

The mycobacteria genus is a group of acid-fast species characterized by a lipid-rich waxy cell wall much thicker than that found in most other bacteria. Notable members of the genus include Mycobacterium tuberculosis, Mycobacterium leprae, and Mycobacterium ulcerans, causative agents of tuberculosis (TB), leprosy and Buruli ulcers, respectively. In addition, numerous other nontuberculosis mycobacteria (NTM) cause various diseases including pulmonary and skin infections.

Lipids in the cell envelope of mycobacteria typically make up 30-60\% of the dry weight and are thought to play important biological roles, notably in determining virulence and drug resistance [1]. While the importance of lipids in mycobacterial infections has been known for many years, there has been a recent renaissance in the study of lipids in these bacteria. This can be ascribed to the emergence of the fields of lipidomics and systems biology driven by technical advances in mass spectrometry (MS) instrumentation. In particular, the use of atmospheric pressure ionization (especially electrospray ionization, ESI) and the coupling of liquid chromatography (LC) to MS have allowed the identification and quantitation of ever-increasing numbers of lipid species.

Although lipid metabolism in the whole genus of mycobacteria is of interest, much recent research has been focused on M. tuberculosis, and closely-related species, due to the huge burden on global health caused by TB. It is estimated that approximately one third of the human population are latently infected with $M$. tuberculosis despite improvements in public health and the discovery of effective chemotherapeutic treatments [2]. The problem has been aggravated by the emergence of multidrugresistant (MDR) and extensively drug-resistant (XDR) M. tuberculosis strains that are increasingly 
difficult to treat, along with co-incidence of $\mathrm{TB}$ with other diseases, including human immunodeficiency virus (HIV) infection.

In this review, we provide an overview of recent investigations into the lipid metabolism of mycobacteria using MS to determine metabolite structures and analyze the biological effects of altered lipid biochemistry. The work described here is not an exhaustive summary of mycobacterial lipidomics, but rather an overview of important advances in the subject in recent years. A summary of the research described is provided in Table 1.

In the first sections, we discuss the targeted identification of individual lipid classes and the use of MS for the quantitation of lipids, structural elucidation by tandem MS, and measurement of lipid biomarkers that may be used to identify an individual species or strain of bacteria. These studies typically use instruments capable of performing tandem MS experiments such as triple quadrupoles and ion traps. We then present global lipidomics and systems biology approaches to the determination of biosynthetic pathways and regulatory networks. Broadly speaking, these are studies that use high resolution MS techniques to analyze multiple lipid classes at the same time. It is important to note that these two lipidomics techniques complement each other, for example novel lipids identified in global approaches can be further investigated by more targeted techniques [3].

\section{Mycolic Acids}

Mycolic acids are a class of fatty acid unique to mycobacteria, and closely-related species, characterized by a long beta-hydroxy chain with a shorter alpha side chain (Figure 1A). In the past, gas chromatography (GC)-MS has been widely used for the analysis of mycolic acids [4]. However, GCMS typically relies on electron ionization (EI) which leads to complex fragmentation patterns that are difficult to interpret. In addition, derivatization of mycolic acids before analysis is necessary to enhance volatility. Mass spectrometry with multistage fragmentation $\left(\mathrm{MS}^{\mathrm{n}}\right)$ using ESI can overcome these problems - mycolic acids form intense ions in negative ion mode MS and collision-induced dissociated (CID) can then be used to determine the structures of the alpha and beta side chains (Figure 2). 
Using ESI-MS ${ }^{\mathrm{n}}, M$. tuberculosis has been shown to contain high levels of mycolic acids in three distinct classes: alpha, keto and methoxy (Figure 1A). In contrast, Mycobacterium bovis Bacillus Calmette Guérin (BCG) Pasteur contains only very low levels of methoxy-mycolic acids [5]. NTM and related species also produce mycolic acids, but can be distinguished from M. tuberculosis by the length of the carbon chains present [6]. For example, bacteria of the Rhodococcus family synthesize mycolic acids with an overall carbon count of around 30-50 (c.f. > 60 in M. tuberculosis). Rhodococcus equi was shown to produce hundreds of different mycolic acid structures with alpha chains containing 10-18 carbons. This is in stark contrast to mycobacteria species which typically produce longer alpha chains of just one or two carbon chain lengths, for example C-22 and C-24 in $M$. tuberculosis. Such detailed analysis of the molecular makeup of mycolic acids was made possible by using $\mathrm{MS}^{\mathrm{n}}$ which could detect up to 10 distinct isobaric molecular species for a single mass. This demonstrates both the complexity of lipid biochemistry and the power of modern analytical techniques $[7,8]$.

The high resolution technology has enabled studies to address diverse important biological questions. For instance, it was demonstrated that the mycolic acid profile of M. tuberculosis can change over time in response to growth conditions. A subpopulation of mycolic acids, specifically some alphamycolic acids, were detected at high levels in bacteria grown under hypoxic conditions, but at much lower levels after aeration of the cultures. Conversely, several species of keto-mycolic acids were almost entirely eliminated during oxygen deprivation, but increased in concentration after return to normoxic conditions [5]. Growth temperature can also have an effect on the precise mycolic acid content of organisms - Corynebacterium glutamicum was shown to produce mycolic acids with shorter carbon chains and a greater degree of unsaturation when grown at $34{ }^{\circ} \mathrm{C}$ compared to $30{ }^{\circ} \mathrm{C}$ [9].

Resolving the fine details of mycolic acids is instrumental as differences in mycolic acid content may have important roles in mycobacterial disease and treatment. For example, an $M$. bovis BCG strain lacking the ethA-ethR locus had lower overall mycolic acid levels, with a marked decrease in those with C-24 alpha chains [10]. As the ethA-ethR locus is vital for the activation of the chemotherapeutic 
agent ethionamide, a better understanding of its biological role and functional implication of mycolic acid alterations is important in the context of the development of drug resistance.

In the past, the field of chemotaxonomy has capitalized on the presence of mycolic acids unique to $M$. tuberculosis to develop diagnostic tests for TB using high performance liquid chromatography (HPLC) with ultraviolet (UV) detection of derivatized mycolic acids [11]. While these methods are able to differentiate mycobacteria, the additional structural information and sensitivity offered by MS can offer significant improvements. For example, LC-MS has been used to determine the structure of mycolic acids detected in a classic normal-phase HPLC assay of mycolic acid methyl esters enabling the differentiation of 13 M. bovis substrains [12].

More recently, a method was described to successfully detect mycolic acids in sputum samples taken from patients with suspected TB. In this method, a synthetic mycolic acid standard was added to the samples to allow accurate quantification of the individual components. Multiple reaction monitoring (MRM) was used to analyze around 2000 compounds across 33 species of mycobacteria to generate a very detailed map of mycolic acid content. Using this information, a diagnostic test for TB was developed with $94 \%$ sensitivity and $93 \%$ specificity, comparable to the widely used smear test [13]. The absence of a culture step is a major advantage of this assay, although 10 days of culture prior to analysis has been shown to significantly improve sensitivity in a similar method [14].

In a related application, MS was used to detect mycolic acids in archeological bone samples. Fourteen mycolic acids were detected in a skeleton from the Neolithic period with bone lesions characteristic of skeletal TB. In this case, identification of lipids unique to M. tuberculosis offered extra evidence of TB infection to complement diagnoses based on anatomical features and analysis of bacterial DNA [15].

\section{Glycolipids}

Glycolipids have long been known to be present in M. tuberculosis, with mannose and inositol first identified in a phospholipid extract of M. tuberculosis in 1930 [16, 17]. Later work determined the 
structures of phosphatidyl-myo-inositol mannosides (PIMs, Figure 1B) incorporating 1 to 6 mannose units with several fatty acid substituents, potentially giving rise to hundreds of individual species [18].

More recently, $\mathrm{MS}^{\mathrm{n}}$ on a linear ion trap (LIT) has been used for the structural determination of PIMs isolated from M. bovis BCG. Characteristic fragments in the $\mathrm{MS}^{3}$ spectra allowed the assignment of the position of fatty acids on the carbohydrate backbone of around 20 distinct molecular species [19]. By extending the analysis to include $\mathrm{MS}^{4}$ experiments, over 100 additional PIMs were identified incorporating one or two more fatty acid substituents on the glycoside core [20].

A second class of glycolipid in mycobacteria incorporates a trehalose unit substituted with one or more mycolate side chains (Figure 1C). Trehalose monomycolates (TMMs) and dimycolates (TDMs) were isolated from Mycobacterium simiae using a method involving multiple stages of chromatography. While the approximate structures of the isolated lipids were revealed using nuclear magenetic resonance (NMR) spectroscopy, MS allowed a much more detailed analysis of the metabolites [21]. Hydrolysis released free mycolic acids which were analysed by MS to reveal the distinct molecular species, however, as no $\mathrm{MS}^{\mathrm{n}}$ experiments was performed, only the total carbon count of the mycolic acids was determined, with no information on the length of each carbon chain.

A simplified method for TMM/TDM analysis was developed based on a rapid solid phase extraction (SPE) of the intact glycolipids followed by $\mathrm{MS}^{\mathrm{n}}$ in a LIT to determine the exact structure [22]. The fragmentation patterns revealed the make-up of the mycolate chains, including the structure of the alpha- and beta-hydroxy branches. This method was used to identify hundreds of species of TMM and TDM in $R$. equi, revealing the complexity of this class of lipids. Other MS experiments have demonstrated the requirement of acetylation of TMMs for their transport to the outer membrane in $C$. glutamicum, which has potential implications for new and current drug targets in mycobacteria species [23].

Additional classes of glycolipids have been identified that are made up of trehalose along with several acyl chains. These groups include diacyl trehaloses (DATs), triacyl trehaloses (TATs) and pentaacyl trehaloses (PATs) (Figure 1D) [24]. All of these metabolites incorporate mycosanoic or mycolipanolic 
acids in place of the mycolic acids found in TMMs and TDMs, and are related to the sulfated glycolipids discussed in the following section.

Other glycolipids may also play important roles in M. tuberculosis virulence and drug susceptibility. For example, MS was used to show that a Mycobacterium marinum strain deficient in tesA produced only trace amounts of phenolic glycolipids (PGLs, Figure 1E) but had a slight increase in growth yield. Interestingly, the knockout strain demonstrated hypersusceptibility to several antibiotics suggesting that these lipids play an important role in the mycobacterial response to chemotherapeutic agents [25].

MS is particularly useful for identifying subtle differences in lipid biochemistry that can nevertheless influence infection and the immune response. A mannosyl $\beta$-1-phosphoisoprenoid (MPI, Figure 1F) with a carbohydrate group identical to the mammalian mannosyl $\beta$-1-phosphodolichol (MPD) but an unusual branched lipid attachment was identified using LC-MS [26]. Biochemical studies then demonstrated that this compound was necessary for the activation of a subset of T-cells and identified pks 12 as a candidate gene predicted to catalyse several key steps in the biosynthesis of this lipid class. This presents a good example of data from MS studies directing further biochemical experiments leading to a detailed understanding of lipid biology.

\section{Sulfolipids}

MS is clearly a useful tool to confirm the structures of previously described lipids and identify new metabolites. For example, the sulfated glycolipids (SGLs) of the class sulfolipid-I (SL-I, Figure 1G) along with several other sulfur-containing metabolites were identified in $M$. tuberculosis and Mycobacterium smegmatis by using a growth media incorporating a source of ${ }^{34} \mathrm{~S}$-labelled sulfate [27]. High resolution-accurate mass (HRAM) MS revealed metabolites differing by two mass units, corresponding to those incorporating ${ }^{32} \mathrm{~S}$ and ${ }^{34} \mathrm{~S}$.

Further insights into the metabolism of SL-I were gleaned from experiments carried out using a strain of M. tuberculosis lacking a putative ATP binding cassette (ABC) transporter known as mce2 [28]. MS analysis revealed an accumulation of SL-I and a biosynthetic precursor, $\mathrm{SL}_{1278}$, suggesting that the 
role of mce 2 is to transport SL-I across the bacterial cell wall, where it may alter the structure of the lipid envelope during the course of infection.

Additional MS ${ }^{\mathrm{n}}$ experiments using lipids extracted from M. tuberculosis (H37Rv strain) have provided detailed structural information for SL-I and SL-II, and further demonstrated that SL-II is the major class of mycobacterial sulfolipid rather than SL-I as had previously been thought [29].

\section{Triacylglycerols and related lipids}

An important trait for the success of M. tuberculosis is the ability of the organism to survive for prolonged periods in a dormant state in hypoxic conditions in granulomas in the lungs of the host. As part of this response, M. tuberculosis accumulates triacylglycerols (TAGs) under hypoxic conditions [30]. Interestingly, strains from the W/Beijing family have been shown to accumulate TAGs when grown in normoxic conditions, which was proposed to relate to the high virulence and widespread drug resistance of these lineages [31].

MS analysis of M. bovis BCG cultures have shown that this organism also accumulates TAGs during hypoxia, before extensive degradation on re-aeration [32]. This breakdown of TAGs was shown to correspond to an increase in TAG lipase activity, and the utilization of TAGs after reaeration was blocked by using the anti-obesity drug tetrahydrolipistatin (THL) which inhibits these enzymes [33].

$\mathrm{MS}^{\mathrm{n}}$ experiments have been used to determine the exact structure of TAGs and the closely related monomeromycolyl diacylglycerols (mmDAGs, Figure 1H) in M. smegmatis [34]. In both TAGs and mmDAGs the $s n-1$ position was found to be almost exclusively substituted with FA 18:1 while the $s n$ 2 position varied between FA 16:0 and 16:1. The $s n-3$ substituent was far more variable, with various fatty acids (in the case of TAGs) or meromycolic acids (in mmDAGs) filling this position.

\section{Phthiocerol dimycocerosates}

Phthiocerol dimycocerosates (PDIMs, Figure 1I) are another class of polyacylated lipid found in mycobacteria species. These important waxes are related to PGLs and were shown to follow the same reduction in abundance as PGLs in a tesA deficient strain of $M$. marinum [25]. PDIMs bind to mycolic 
acids to form the outer layer of the cell envelope along with other multi-methyl branched lipids such as DATs, PATs, and SGLs [24]. In addition to their important biological roles, PDIMs may be extremely useful for the detection and identification of mycobacteria species. For example, a GC-MS method has been developed that could diagnose M. tuberculosis infection from sputum samples with $65 \%$ sensitivity and $76 \%$ specificity [35]. In principle, a combination of this method with other lipidomics techniques described here, such as MA analysis, may lead to a powerful diagnostic tool based solely on lipid MS.

PDIMs have also proven to be useful for the detection of mycobacteria species from ancient samples. Mycolic acids were detected in two samples from a 17,000 year old bison skeleton, but due to degradation, they could not conclusively be linked to $M$. tuberculosis. However, both the mycocerosate and phthiocerol components of PDIMs were identified by GC-MS, providing conclusive proof of tuberculosis infection [36].

\section{Global lipidomics of mycobacteria}

The examples given above discuss methods designed to measure a specific lipid, or class of lipids, but there is a growing trend in lipidomics towards more global analyses of lipidomes. In a typical global analysis, identification of lipids is based largely on mass-to-charge ratio $(\mathrm{m} / \mathrm{z})$, thus, the instruments used for these studies are necessarily those capable of recording spectra at high-resolution (HR) with accurate mass $(\mathrm{AM})$ detection. $\mathrm{MS}^{\mathrm{n}}$ experiments are also important for providing additional information to support the assignment of structures.

In any global lipidomics analysis, it is important to consider the diverse chemical properties of lipids, as this will drastically affect the ionization efficiency of different compounds leading to an overrepresentation of some metabolites. Also, it is inevitable that some lipid species of very low abundance will be missed due to the inherently limited dynamic range of the instruments used. The array of unusual lipids found in mycobacteria species presents an additional challenge, requiring the development of new databases that incorporate these metabolites. 
In an early example of a global approach in $M$. tuberculosis, extracted lipids were infused into a Fourier transform ion cyclotron resonance (FT-ICR) mass spectrometer to generate a raw data set of over 1800 ions [37]. The raw data was analyzed using an in-house developed algorithm, named the fatty acid analysis tool (FAAT), which creates a list of molecular features that can be assigned lipid structures by comparison to a customizable database. Applying this approach, a wild-type Erdman strain of $M$. tuberculosis was compared to a mutant lacking the mmpl8 gene, coding for an enzyme involved in the biosynthesis of the SL-I class of lipid. This revealed an accumulation of an intermediate in the SL-I biosynthesis pathway in the mutant strain.

In a similar experiment, lipids of several strains of M. tuberculosis with mutations in genes involved in SL-I and PDIM biosynthesis were analyzed [38]. Again, an FT-ICR instrument was employed, and hundreds of putative lipid peaks were identified. As expected, no SL-I peaks were detected in strains lacking enzymes involved in SL-I production, but surprisingly SL-I levels were greatly increased in strains with defective PDIM biosynthesis. As a result, a link between methylmalonyl CoA availability and SL-I and PDIM biosynthesis was observed, demonstrating the power of analyzing a broad array of lipids for the discovery of unexpected correlations that may not be discovered using more targeted approaches.

In a similar vein, a global lipidomics approach involving several extractions and chromatographic methods led to the detection of 12,135 molecular features (MFs) in an M. tuberculosis extract [39]. By focusing on lipids involved in iron homeostasis, an unexpected dideoxymycobactin (DDM) was detected, with the structure confirmed by comparison with a synthetic standard. Analysis of putative intermediates in the biosynthetic pathway led to the proposal of a revised route to these metabolites, with experiments using knockout strains lending credence to the model.

More recently, attempts have been made to create a lipid database containing a significant fraction of mycobacterial lipids. One such method used an LC-MS protocol to separate the major classes of mycobacterial lipids, and therefore detect a wide range of metabolites present at a range of concentrations. In total, 1916 molecular features were obtained from a positive ion mode analysis, and a further $744 \mathrm{MFs}$ in negative ion mode [40]. This data was used to construct a database of 
mycobacterial lipids named Mtb LipidDB with lipid structures broadly characterized based on the criteria set out by the LIPID MAPS consortium [41, 42], but importantly with the addition of a number of new classes and subclasses to take into account lipids unique to mycobacteria. In a proof-ofprinciple biological experiment, abundances of TAGs were shown to vary significantly in different growth phases of M. tuberculosis.

To facilitate the use of these techniques, a freely available software package named MS-LAMP has been developed that provides a graphical user interface (GUI) and search tool that can be used with both the LIPID MAPS database and Mtb LipidDB [43]. To demonstrate the utility of this tool, differences in lipid composition between M. tuberculosis cultures grown at $\mathrm{pH} 5.5$ and those grown at pH 7 were analyzed.

Another database named Mycomass has been developed containing over 5000 lipid entries again classified using a modification of the LIPID MAPS system [44]. A normal phase HPLC method was developed that efficiently separates lipids extracted from mycobacteria. This method was applied to a comparative study of three mycobacteria: the $M$. tuberculosis reference strain $\mathrm{H} 37 \mathrm{Rv}$, the $M$. tuberculosis strain HN878 from the W/Beijing sublineage, and the avirulent species M. smegmatis. Between the two M. tuberculosis strains, 648 MFs were identified with a fold-change of two or more, while the comparison between M. tuberculosis and M. smegmatis revealed 4339 such differences. Further analysis of the data showed that phenolic glycolipids (PGLs) were more abundant in the W/Beijing strain, which is in agreement with previously published genome data [45]. Using knowledge generated from this global approach, a straightforward targeted method was developed that could potentially be used to identify W/Beijing strains in a clinical setting.

Another interesting use of this database was for the identification of lipids that may play an important role in the virulence of mycobacteria species by comparison of the global lipidome of M. tuberculosis H37Rv with M. bovis BCG [46]. In total, 7852 MFs were identified, with 1845 significantly increased in one strain or the other. Analysis of the metabolites with the highest fold-change ratios was followed by large scale culture and chromatographic isolation of one lipid, allowing NMR studies to be carried out to confirm the structure as 1-tuberculosinyladenosine (1-TbAd), a novel diterpene nucleoside. 
Screening 4196 M. tuberculosis mutants led to the discovery that the biosynthesis of 1-TbAd requires the enzyme encoded by Rv3378c. Partial synthesis of 1-TbAd along with overexpression of Rv3378c suggested that this enzyme acts as a tuberculosinyl transferase, possibly with a second binding site that binds adenosine during the catalytic process.

\section{Systems biology approaches incorporating lipidomics}

The lipidomics experiments discussed here clearly provide powerful tools for the analysis of the diverse array of mycobacterial lipids. However, even more information may be available if these tools are incorporated into systems biology experiments that include other "omics" approaches to build up a more complete picture of $M$. tuberculosis metabolism.

A systems-wide analysis of $M$. tuberculosis incorporating transcriptomic, proteomic, metabolomic and lipidomic analyses has recently been reported using a high-throughput chromatin immunoprecipitation-sequencing (ChIP-Seq) method to assay the interactions of 50 transcription factors (TFs) and build up a map of the M. tuberculosis regulatory network [47]. Although the functions of many of these transcription factors were already known, the links between them were not, and this approach showed direct connections between the hypoxic response and lipid metabolism. To validate the network, a hypoxia/re-aeration experiment was carried out using the H37Rv laboratory strain of M. tuberculosis. Gene expression was measured at a number of time-points and generally agreed well with the predicted values. Using a previously developed method [44] lipids were measured before, during and after hypoxia. As expected, hypoxia caused an accumulation of TAGs, with a concurrent decrease in the level of diacylglycerols (DAGs). Analysis of the regulatory network suggested that these changes are due to a down-regulation of DAG production, with the increase in TAGs due to the metabolism of pre-existing DAGs. Changes in the methylmalonyl CoA pathway were also observed, with genes involved in the biosynthesis of SGLs, PATs, DATs and PDIMs all downregulated. The lipidomic data supported this observation with SGL, PAT and DAT levels static, and a decrease in PDIM concentrations. The mycolic acid content also varied at the different time points with an increase in free mycolates during hypoxia followed by a decrease on re-aeration. The metabolism of TMMs and TDMs followed the opposite pattern, suggesting that mycolic acids are 
cleaved from trehalose-containing lipids during hypoxia. However, as the MS data used was acquired in positive ion mode, only two mycolic acids were reported highlighting the difficulty of performing a global lipidomic analysis in a single experiment.

More recently, a combined genomics and lipidomics approach for the analysis of 35 clinical $M$. tuberculosis strains from four distinct lineages was reported [48]. In this study, a phylogenetic tree constructed using only mutations found in proteins thought to be involved in mycolic acid metabolism closely matched a tree based on full genome data, suggesting that the genetic diversity of $M$. tuberculosis strains may be mirrored in their mycolic acid metabolism. To test this hypothesis, 80 mycolic acids were analysed by MS using a previously described targeted method [13]. The metabolism of oxygenated mycolic acids was shown to be significantly different between ancient and modern lineages and more subtle differences such as a low level of alpha C-24 mycolic acids in lineage 6 strains was observed. A computational approach was used to predict the functional effects of mutations in mycolic acid related proteins, with the majority of mutations that were predicted to alter activity belonging to strains from the ancient lineages. These subtle differences in mycolic acid metabolism may play an important role in the varied susceptibility of M. tuberculosis strains to antimycobacterial therapies.

\section{Concluding remarks}

The work presented here demonstrates the power of MS-based lipidomics experiments for the discovery, identification and quantification of lipids in mycobacteria and their applications to unravel novel functions of these metabolites. Using these approaches, mycobacterial infections can be diagnosed, novel and unusual lipids discovered, and important differences between organisms uncovered.

In the future, developments in MS and chromatography instrumentation will allow the profiling of more lipids with greater accuracy than ever before. In addition, novel techniques will allow more detailed studies of specific lipids and their interactions with other biomolecules. For example, ion mobility (IM) coupled to mass spectrometry has already been used to demonstrate that a mechanosensitive mycobacterial membrane protein (mechano-sensitive channel of large conductance, $\mathrm{MscL}$ ) is 
stabilized by phosphatidylinositol phosphate, suggesting a role for this lipid in modulating protein activity [49].

The studies described in this review were based on whole bacteria and complementing with other biochemical techniques will allow the subcellular distribution of lipids to be determined, giving more detailed insights into their biological importance. Indeed, a technique has been developed that effectively separates lipids present in the outer membrane from those in the inner membrane allowing the analysis of different components of the cell [50].

Just as important as advances in instrumentation will be the development of larger databases of the unique lipids found in mycobacteria along with new data processing software and search tools. Lipidomics data will be integrated with systems biology models of mycobacterial metabolism and regulation as clearly demonstrated by various studies $[47,48]$.

Taken together, these advances will lead to a detailed understanding of mycobacterial lipid metabolism and the roles these biomolecules play in infection and drug resistance. Ultimately, this will provide information that can be used to direct the development of new therapies for diseases caused by Mycobacterium species.

\section{Acknowledgments}

Works in the group of X.L.G. were supported by grants from the Swiss National Science Foundation (Ambizione, PZ00P3_136738) and SystemsX.ch (TbX).

\section{References}

[1] M. Daffé, P. Draper, The envelope layers of mycobacteria with reference to their pathogenicity, Adv Microb Physiol, 39 (1998) 131-203.

[2] Global Tuberculosis Report, in, World Health Organisation, Geneva, 2014.

[3] X.L. Guan, M.R. Wenk, Targeted and non-targeted analysis of membrane lipids using mass spectrometry, Methods Cell Biol, 108 (2012) 149-172. 
[4] K. Kaneda, S. Naito, S. Imaizumi, I. Yano, S. Mizuno, I. Tomiyasu, T. Baba, E. Kusunose, M. Kusunose, Determination of molecular species composition of C80 or longer-chain alpha-mycolic acids in Mycobacterium spp. by gas chromatography-mass spectrometry and mass chromatography, $\mathbf{J}$ Clin Microbiol, 24 (1986) 1060-1070.

[5] G. Shui, A.K. Bendt, K. Pethe, T. Dick, M.R. Wenk, Sensitive profiling of chemically diverse bioactive lipids, J Lipid Res, 48 (2007) 1976-1984.

[6] S.H. Song, K.U. Park, J.H. Lee, E.C. Kim, J.Q. Kim, J. Song, Electrospray ionization-tandem mass spectrometry analysis of the mycolic acid profiles for the identification of common clinical isolates of mycobacterial species, J Microbiol Methods, 77 (2009) 165-177.

[7] F.F. Hsu, K. Soehl, J. Turk, A. Haas, Characterization of mycolic acids from the pathogen Rhodococcus equi by tandem mass spectrometry with electrospray ionization, Anal Biochem, 409 (2011) 112-122.

[8] I. Kolouchova, O. Schreiberova, J. Masak, K. Sigler, T. Rezanka, Structural analysis of mycolic acids from phenol-degrading strain of Rhodococcus erythropolis by liquid chromatography-tandem mass spectrometry, Folia Microbiol (Praha), 57 (2012) 473-483.

[9] Y. Yang, F. Shi, G. Tao, X. Wang, Purification and structure analysis of mycolic acids in Corynebacterium glutamicum, J Microbiol, 50 (2012) 235-240.

[10] M.L. Ang, Z.Z. Siti, G. Shui, P. Dianiskova, J. Madacki, W. Lin, V.H. Koh, J.M. Martinez Gomez, S. Sudarkodi, A. Bendt, M. Wenk, K. Mikusova, J. Kordulakova, K. Pethe, S. Alonso, An ethA-ethR-deficient Mycobacterium bovis BCG mutant displays increased adherence to mammalian cells and greater persistence in vivo, which correlate with altered mycolic acid composition, Infect Immun, 82 (2014) 1850-1859.

[11] W.R. Butler, L.S. Guthertz, Mycolic Acid Analysis by High-Performance Liquid Chromatography for Identification of Mycobacterium Species, Clin Microbiol Rev, 14 (2001) 704726.

[12] Y. Uenishi, T. Takii, I. Yano, M. Sunagawa, Separation and molecular characterization of mycolic acid from the cell wall skeleton of Mycobacterium bovis BCG Tokyo 172 (SMP-105) and 
BCG substrains by normal-phase high performance liquid chromatography and liquid chromatography/mass spectrometry, J Microbiol Methods, 77 (2009) 320-322.

[13] G. Shui, A.K. Bendt, I.A. Jappar, H.M. Lim, M. Laneelle, M. Hervé, L.E. Via, G.H. Chua, M.W. Bratschi, S.Z. Zainul Rahim, A.L. Michelle, S.H. Hwang, J.S. Lee, S.Y. Eum, H.K. Kwak, M. Daffé, V. Dartois, G. Michel, C.E. Barry, 3rd, M.R. Wenk, Mycolic acids as diagnostic markers for tuberculosis case detection in humans and drug efficacy in mice, EMBO Mol Med, 4 (2012) 27-37.

[14] R. Szewczyk, K. Kowalski, B. Janiszewska-Drobinska, M. Druszczynska, Rapid method for Mycobacterium tuberculosis identification using electrospray ionization tandem mass spectrometry analysis of mycolic acids, Diagn Microbiol Infect Dis, 76 (2013) 298-305.

[15] B. Borowska-Strugiń, M. Druszczyńska, W. Lorkiewicz, R. Szewczyk, E. Ządzińska, Mycolic Acids as Markers of Osseous Tuberculosis in the Neolithic Skeleton from Kujawy Region (Central Poland), in: Anthropological Review, vol. 77, 2014, pp. 137.

[16] R.J. Anderson, The chemistry of the lipoids of tubercle bacilli. XIV. The occurance of inosite in the phosphatide from human tubercle bacilli, J Am Chem Soc, 52 (1930) 1607-1608.

[17] R.J. Anderson, A.G. Renfrew, The chemistry of the lipoids of tubercle bacilli. XIII. The occurance of mannose in the phosphatide from human tubercle bacilli, J Am Chem Soc, 52 (1930) $1252-1254$.

[18] Y.C. Lee, C.E. Ballou, Complete structures of the glycophospholipids of mycobacteria, Biochemistry, 4 (1965) 1395-1404.

[19] F.F. Hsu, J. Turk, M. Owens Róí, E.R. Rhoades, D.G. Russell, Structural Characterization of Phosphatidyl-myo-inositol Mannosides from Mycobacterium bovis Bacillus Calmette Guérin by Multiple-Stage Quadrupole Ion-Trap Mass Spectrometry with Electrospray Ionization. I. PIMs and Lyso-PIMs, J Am Soc Mass Spectrom, 18 (2007) 466-478.

[20] F.F. Hsu, J. Turk, M. Owens Róí, E.R. Rhoades, D.G. Russell, Structural Characterization of Phosphatidyl-myo-Inositol Mannosides from Mycobacterium bovis Bacillus Calmette Gúerin by Multiple-Stage Quadrupole Ion-Trap Mass Spectrometry with Electrospray Ionization. II. Monoacyland Diacyl-PIMs, J Am Soc Mass Spectrom, 18 (2007) 479-492. 
[21] L.M. Mederos, E.H. Montoro, A. Bernabeu, C. Linares, P.L. Valero-Guillen, Structural studies of cord factors from Mycobacterium simiae related to the capacity for tumour necrosis factor alpha (alpha-TNF) induction, Microbiology, 156 (2010) 3744-3753.

[22] F.F. Hsu, J. Wohlmann, J. Turk, A. Haas, Structural definition of trehalose 6-monomycolates and trehalose 6,6'-dimycolates from the pathogen Rhodococcus equi by multiple-stage linear ion-trap mass spectrometry with electrospray ionization, J Am Soc Mass Spectrom, 22 (2011) 2160-2170.

[23] Y. Yamaryo-Botte, A.K. Rainczuk, D.J. Lea-Smith, R. Brammananth, P.L. van der Peet, P. Meikle, J.E. Ralton, T.W. Rupasinghe, S.J. Williams, R.L. Coppel, P.K. Crellin, M.J. McConville, Acetylation of Trehalose Mycolates Is Required for Efficient MmpL-Mediated Membrane Transport in Corynebacterineae, ACS Chem Biol, 10 (2015) 734-746.

[24] D.E. Minnikin, L. Kremer, L.G. Dover, G.S. Besra, The methyl-branched fortifications of Mycobacterium tuberculosis, Chem Biol, 9 (2002) 545-553.

[25] S.S. Chavadi, U.R. Edupuganti, O. Vergnolle, I. Fatima, S.M. Singh, C.E. Soll, L.E. Quadri, Inactivation of tesA reduces cell wall lipid production and increases drug susceptibility in mycobacteria, J Biol Chem, 286 (2011) 24616-24625.

[26] I. Matsunaga, A. Bhatt, D.C. Young, T.Y. Cheng, S.J. Eyles, G.S. Besra, V. Briken, S.A. Porcelli, C.E. Costello, W.R. Jacobs, Jr., D.B. Moody, Mycobacterium tuberculosis pks12 produces a novel polyketide presented by CD1c to T cells, J Exp Med, 200 (2004) 1559-1569.

[27] J.D. Mougous, M.D. Leavell, R.H. Senaratne, C.D. Leigh, S.J. Williams, L.W. Riley, J.A. Leary, C.R. Bertozzi, Discovery of sulfated metabolites in mycobacteria with a genetic and mass spectrometric approach, Proc Natl Acad Sci U S A, 99 (2002) 17037-17042.

[28] O. Marjanovic, A.T. Iavarone, L.W. Riley, Sulfolipid accumulation in Mycobacterium tuberculosis disrupted in the mce2 operon, J Microbiol, 49 (2011) 441-447.

[29] E.R. Rhoades, C. Streeter, J. Turk, F.F. Hsu, Characterization of Sulfolipids of Mycobacterium tuberculosis H37Rv by Multiple-stage Linear Ion-trap High Resolution Mass Spectrometry with Electrospray Ionization Reveals that Family of Sulfolipid II predominates, Biochemistry, 50 (2011) 9135-9147. 
[30] J. Daniel, C. Deb, V.S. Dubey, T.D. Sirakova, B. Abomoelak, H.R. Morbidoni, P.E. Kolattukudy, Induction of a Novel Class of Diacylglycerol Acyltransferases and Triacylglycerol Accumulation in Mycobacterium tuberculosis as It Goes into a Dormancy-Like State in Culture, J Bacteriol, 186 (2004) 5017-5030.

[31] M.B. Reed, S. Gagneux, K. Deriemer, P.M. Small, C.E. Barry, 3rd, The W-Beijing lineage of Mycobacterium tuberculosis overproduces triglycerides and has the DosR dormancy regulon constitutively upregulated, J Bacteriol, 189 (2007) 2583-2589.

[32] K.L. Low, P.S.S. Rao, G. Shui, A.K. Bendt, K. Pethe, T. Dick, M.R. Wenk, Triacylglycerol Utilization Is Required for Regrowth of In Vitro Hypoxic Nonreplicating Mycobacterium bovis Bacillus Calmette-Guerin, J Bacteriol, 191 (2009) 5037-5043.

[33] M.S. Ravindran, S.P. Rao, X. Cheng, A. Shukla, A. Cazenave-Gassiot, S.Q. Yao, M.R. Wenk, Targeting lipid esterases in mycobacteria grown under different physiological conditions using activity-based profiling with tetrahydrolipstatin (THL), Mol Cell Proteomics, 13 (2014) 435-448.

[34] G.E. Purdy, S. Pacheco, J.T. and, F.F. Hsu, Characterization of mycobacterial triacylglycerols and monomeromycolyl diacylglycerols from Mycobacterium smegmatis biofilm by electrospray ionization multiple-stage and high-resolution mass spectrometry, Anal Bioanal Chem, 405 (2013) 7415-7426.

[35] D.M. O'Sullivan, S.C. Nicoara, R. Mutetwa, S. Mungofa, O.Y. Lee, D.E. Minnikin, M.W. Bardwell, E.L. Corbett, R. McNerney, G.H. Morgan, Detection of Mycobacterium tuberculosis in sputum by gas chromatography-mass spectrometry of methyl mycocerosates released by thermochemolysis, PLoS One, 7 (2012) e32836.

[36] O.Y. Lee, H.H. Wu, H.D. Donoghue, M. Spigelman, C.L. Greenblatt, I.D. Bull, B.M. Rothschild, L.D. Martin, D.E. Minnikin, G.S. Besra, Mycobacterium tuberculosis complex lipid virulence factors preserved in the 17,000-year-old skeleton of an extinct bison, Bison antiquus, PLoS One, 7 (2012) e41923.

[37] M.D. Leavell, J.A. Leary, Fatty acid analysis tool (FAAT): An FT-ICR MS lipid analysis algorithm, Anal Chem, 78 (2006) 5497-5503. 
[38] M. Jain, C.J. Petzold, M.W. Schelle, M.D. Leavell, J.D. Mougous, C.R. Bertozzi, J.A. Leary, J.S. Cox, Lipidomics reveals control of Mycobacterium tuberculosis virulence lipids via metabolic coupling, Proc Natl Acad Sci U S A, 104 (2007) 5133-5138.

[39] C.A. Madigan, T.Y. Cheng, E. Layre, D.C. Young, M.J. McConnell, C.A. Debono, J.P. Murry, J.R. Wei, C.E. Barry, 3rd, G.M. Rodriguez, I. Matsunaga, E.J. Rubin, D.B. Moody, Lipidomic discovery of deoxysiderophores reveals a revised mycobactin biosynthesis pathway in Mycobacterium tuberculosis, Proc Natl Acad Sci U S A, 109 (2012) 1257-1262.

[40] M.J. Sartain, D.L. Dick, C.D. Rithner, D.C. Crick, J.T. Belisle, Lipidomic analyses of Mycobacterium tuberculosis based on accurate mass measurements and the novel "Mtb LipidDB", J Lipid Res, 52 (2011) 861-872.

[41] E. Fahy, S. Subramaniam, H.A. Brown, C.K. Glass, A.H. Merrill, Jr., R.C. Murphy, C.R. Raetz, D.W. Russell, Y. Seyama, W. Shaw, T. Shimizu, F. Spener, G. van Meer, M.S. VanNieuwenhze, S.H. White, J.L. Witztum, E.A. Dennis, A comprehensive classification system for lipids, J Lipid Res, 46 (2005) 839-861.

[42] E. Fahy, S. Subramaniam, R.C. Murphy, M. Nishijima, C.R.H. Raetz, T. Shimizu, F. Spener, G. van Meer, M.J.O. Wakelam, E.A. Dennis, Update of the LIPID MAPS comprehensive classification system for lipids, J Lipid Res, 50 (2009) S9-S14.

[43] V. Sabareesh, G. Singh, Mass spectrometry based lipid(ome) analyzer and molecular platform: a new software to interpret and analyze electrospray and/or matrix-assisted laser desorption/ionization mass spectrometric data of lipids: a case study from Mycobacterium tuberculosis, J Mass Spectrom, 48 (2013) 465-477.

[44] E. Layre, L. Sweet, S. Hong, C.A. Madigan, D. Desjardins, D.C. Young, T.Y. Cheng, J.W. Annand, K. Kim, I.C. Shamputa, M.J. McConnell, C.A. Debono, S.M. Behar, A.J. Minnaard, M. Murray, C.E. Barry, I. Matsunaga, D.B. Moody, A comparative lipidomics platform for chemotaxonomic analysis of Mycobacterium tuberculosis, Chem Biol, 18 (2011) 1537-1549.

[45] P. Constant, E. Perez, W. Malaga, M.A. Laneelle, O. Saurel, M. Daffé, C. Guilhot, Role of the pks15/1 gene in the biosynthesis of phenolglycolipids in the Mycobacterium tuberculosis complex. Evidence that all strains synthesize glycosylated p-hydroxybenzoic methyl esters and that strains 
devoid of phenolglycolipids harbor a frameshift mutation in the pks15/1 gene, J Biol Chem, 277 (2002) 38148-38158.

[46] E. Layre, H.J. Lee, D.C. Young, A.J. Martinot, J. Buter, A.J. Minnaard, J.W. Annand, S.M. Fortune, B.B. Snider, I. Matsunaga, E.J. Rubin, T. Alber, D.B. Moody, Molecular profiling of Mycobacterium tuberculosis identifies tuberculosinyl nucleoside products of the virulence-associated enzyme Rv3378c, Proc Natl Acad Sci U S A, 111 (2014) 2978-2983.

[47] J.E. Galagan, K. Minch, M. Peterson, A. Lyubetskaya, E. Azizi, L. Sweet, A. Gomes, T. Rustad, G. Dolganov, I. Glotova, T. Abeel, C. Mahwinney, A.D. Kennedy, R. Allard, W. Brabant, A. Krueger, S. Jaini, B. Honda, W.H. Yu, M.J. Hickey, J. Zucker, C. Garay, B. Weiner, P. Sisk, C. Stolte, J.K. Winkler, Y. Van de Peer, P. Iazzetti, D. Camacho, J. Dreyfuss, Y. Liu, A. Dorhoi, H.J. Mollenkopf, P. Drogaris, J. Lamontagne, Y. Zhou, J. Piquenot, S.T. Park, S. Raman, S.H. Kaufmann, R.P. Mohney, D. Chelsky, D.B. Moody, D.R. Sherman, G.K. Schoolnik, The Mycobacterium tuberculosis regulatory network and hypoxia, Nature, 499 (2013) 178-183.

[48] D. Portevin, S. Sukumar, M. Coscolla, G. Shui, B. Li, X.L. Guan, A.K. Bendt, D. Young, S. Gagneux, M.R. Wenk, Lipidomics and genomics of Mycobacterium tuberculosis reveal lineagespecific trends in mycolic acid biosynthesis, Microbiologyopen, 3 (2014) 823-835.

[49] A. Laganowsky, E. Reading, T.M. Allison, M.B. Ulmschneider, M.T. Degiacomi, A.J. Baldwin, C.V. Robinson, Membrane proteins bind lipids selectively to modulate their structure and function, Nature, 510 (2014) 172-175.

[50] R. Bansal-Mutalik, H. Nikaido, Mycobacterial outer membrane is a lipid bilayer and the inner membrane is unusually rich in diacyl phosphatidylinositol dimannosides, Proc Natl Acad Sci U S A, 111 (2014) 4958-4963. 
Table 1. Studies using atmospheric pressure ionization techniques for the analysis of lipids from mycobacteria and related species, reviewed in this work. APCI, atmospheric pressure chemical ionization; BCG, Bacillus Calmette Guérin; ESI, electrospray ionization; FT-ICR, Fourier transformion cyclotron resonance; HPLC, high performance liquid chromatography; MALDI, matrix assisted laser desorption/ionization; MS, mass spectrometry $\mathrm{MS}^{\mathrm{n}}$, mass spectrometry with multistage fragmentation; NMR, nuclear magnetic resonance; NTM, non-tuberculosis mycobacteria; Q-TOF, quadrupole-time of flight; Q-trap, quadrupole-ion trap; QqQ, triple quadrupole. Definitions of lipid abbreviations are given in the text and in Figure 1.

\begin{tabular}{|c|c|c|c|c|}
\hline Lipids Analysed & Reference & Organisms & Instruments & Comments \\
\hline \multirow[t]{10}{*}{ Mycolic acids } & {$[5]$} & $\begin{array}{l}\text { M. bovis } \mathrm{BCG} \\
\text { M. tuberculosis }\end{array}$ & ESI-Q-TOF & First ESI analysis of MAs \\
\hline & {$[6]$} & $\begin{array}{l}\text { M. tuberculosis } \\
\text { Various NTM }\end{array}$ & ESI-QqQ & $\begin{array}{l}\text { M. tuberculosis } \\
\text { differentiated from NTM }\end{array}$ \\
\hline & [7] & $\begin{array}{l}\text { R. equi } \\
\text { Rhodococcus } \\
\text { rhodnii }\end{array}$ & $\begin{array}{l}\text { ESI-ion trap } \\
\text { ESI-QqQ }\end{array}$ & $\begin{array}{l}\text { Deuteration used to } \\
\text { confirm structures }\end{array}$ \\
\hline & {$[8]$} & $\begin{array}{l}\text { Rhodococcus } \\
\text { erythropolis }\end{array}$ & ESI-QqQ & $\begin{array}{l}\text { Makeup of MAs varies } \\
\text { with carbon source }\end{array}$ \\
\hline & [9] & $\begin{array}{l}\text { C. glutamicum } \\
\text { (5 strains) }\end{array}$ & ESI-Q-TOF & $\begin{array}{l}\text { Makeup of MAs varies } \\
\text { with growth temperature }\end{array}$ \\
\hline & {$[10]$} & M. bovis $\mathrm{BCG}$ & ESI-Q-trap & $\begin{array}{l}\text { Knockout of ethA-ethR } \\
\text { alters MA makeup }\end{array}$ \\
\hline & {$[12]$} & $\begin{array}{l}\text { M. bovis } \mathrm{BCG} \\
(12 \text { strains })\end{array}$ & APCI-ion trap & $\begin{array}{l}\text { MS used to confirm } \\
\text { HPLC studies }\end{array}$ \\
\hline & {$[13]$} & $\begin{array}{l}\text { M. bovis } \mathrm{BCG} \\
\text { M. tuberculosis } \\
\text { Various NTM }\end{array}$ & $\begin{array}{l}\text { ESI-QqQ } \\
\text { ESI-Q-trap }\end{array}$ & $\begin{array}{l}\text { Sputum samples analysed. } \\
\text { Detailed MA profile of } 33 \\
\text { species. }\end{array}$ \\
\hline & {$[14]$} & M. tuberculosis & ESI-Q-trap & Sputum samples analysed \\
\hline & {$[15]$} & M. tuberculosis & ESI-Q-trap & $\begin{array}{l}\text { MAs identified in } \\
\text { archaeological sample }\end{array}$ \\
\hline \multirow[t]{7}{*}{ Glycolipids } & [19] & M. bovis $\mathrm{BCG}$ & $\begin{array}{l}\text { ESI-ion trap } \\
\text { MALDI-TOF }\end{array}$ & $\begin{array}{l}\text { MS } S^{n} \text { used to identify } \\
\text { structures of PIMs }\end{array}$ \\
\hline & {$[20]$} & M. bovis $\mathrm{BCG}$ & $\begin{array}{l}\text { ESI-ion trap } \\
\text { MALDI-TOF }\end{array}$ & $\begin{array}{l}\text { MS }^{\mathrm{n}} \text { used to identify } \\
\text { structures of PIMs }\end{array}$ \\
\hline & {$[21]$} & M. simiae & ESI-ion trap & $\begin{array}{l}\text { NMR and MS. TMM and } \\
\text { TDM structures. }\end{array}$ \\
\hline & {$[22]$} & R. equi & $\begin{array}{l}\text { ESI-ion trap } \\
\text { ESI-Orbitrap }\end{array}$ & $\begin{array}{l}\text { Determination of TMM } \\
\text { and TDM structures }\end{array}$ \\
\hline & {$[23]$} & C. glutamicum & ESI-Q-trap & $\begin{array}{l}\text { Acetylation is required for } \\
\text { TMM transport }\end{array}$ \\
\hline & {$[25]$} & M. marinum & ESI-Q-TOF & $\begin{array}{l}\text { tes } A \text { mutant deficient in } \\
\text { PDIMs and PGLs }\end{array}$ \\
\hline & {$[26]$} & $\begin{array}{l}\text { M. tuberculosis } \\
\text { M. bovis BCG }\end{array}$ & ESI-ion trap & $\begin{array}{l}\text { MPD lipid product of } \\
p k s 12 \text { activates T-cells }\end{array}$ \\
\hline
\end{tabular}




\begin{tabular}{|c|c|c|c|c|}
\hline \multirow[t]{3}{*}{ Sulfolipids } & [27] & $\begin{array}{l}\text { M. tuberculosis } \\
\text { M. smegmatis }\end{array}$ & ESI-FT-ICR & $\begin{array}{l}\text { Discovery of sulphated } \\
\text { lipids using }{ }^{32} S \text { and }{ }^{34} S\end{array}$ \\
\hline & {$[28]$} & M. tuberculosis & ESI-Q-TOF & $\begin{array}{l}\text { Analysis of mutant } \\
\text { lacking transporter }\end{array}$ \\
\hline & [29] & M. tuberculosis & $\begin{array}{l}\text { ESI-ion trap } \\
\text { ESI-Orbitrap } \\
\text { ESI-FT-ICR }\end{array}$ & $\begin{array}{l}\text { Reassignment of structure } \\
\text { of SL-II }\end{array}$ \\
\hline \multirow[t]{6}{*}{$\begin{array}{l}\text { Triacylglycerols } \\
\text { and related lipids }\end{array}$} & [31] & M. tuberculosis & ESI-quad & $\begin{array}{l}\text { Beijing strains } \\
\text { accumulate TAGs }\end{array}$ \\
\hline & {$[32]$} & M. bovis $\mathrm{BCG}$ & ESI-Q-trap & $\begin{array}{l}\text { TAGs measured across } \\
\text { growth curve }\end{array}$ \\
\hline & {$[33]$} & M. bovis $\mathrm{BCG}$ & ESI-Orbitrap & $\begin{array}{l}\text { TAG metabolism affected } \\
\text { by tetrahydrolipistatin }\end{array}$ \\
\hline & {$[34]$} & M. smegmatis & ESI-Orbitrap & $\begin{array}{l}\text { Structures of TAGs and } \\
\text { mmDAGs }\end{array}$ \\
\hline & {$[35]$} & M. tuberculosis & GC-MS & $\begin{array}{l}\text { PDIM components } \\
\text { detected in sputum }\end{array}$ \\
\hline & [36] & M. tuberculosis & GC-MS & $\begin{array}{l}\text { PDIM components } \\
\text { detected in bison skeleton }\end{array}$ \\
\hline \multirow[t]{7}{*}{ Global Analysis } & {$[37]$} & M. tuberculosis & ESI-FT-ICR & $\begin{array}{l}\text { Software package for data } \\
\text { analysis (FAAT) }\end{array}$ \\
\hline & {$[38]$} & M. tuberculosis & ESI-FT-ICR & $\begin{array}{l}\text { Link between MM-CoA } \\
\text { and SL-I/PDIMs }\end{array}$ \\
\hline & [39] & M. tuberculosis & ESI-Q-TOF & $\begin{array}{l}\text { Revised mycobactin } \\
\text { biosynthesis pathway }\end{array}$ \\
\hline & {$[40]$} & M. tuberculosis & $\begin{array}{l}\text { ESI/APCI- } \\
\text { TOF }\end{array}$ & $\begin{array}{l}\text { Setup of } M t b \text { LipidDB } \\
\text { database }\end{array}$ \\
\hline & {$[43]$} & M. tuberculosis & ESI-Orbitrap & $\begin{array}{l}\text { Search tool for use with } \\
M t b \text { LipidDB }\end{array}$ \\
\hline & [44] & $\begin{array}{l}\text { M. tuberculosis } \\
\text { M. smegmatis }\end{array}$ & ESI-Q-TOF & $\begin{array}{l}\text { Setup of MycoMass and } \\
\text { MycoMap databases }\end{array}$ \\
\hline & {$[46]$} & $\begin{array}{l}\text { M. tuberculosis } \\
\text { M. bovis BCG }\end{array}$ & ESI-Q-TOF & $\begin{array}{l}\text { Novel tuberculosinyl } \\
\text { nucleoside identified }\end{array}$ \\
\hline \multirow[t]{2}{*}{ Systems Biology } & [47] & M. tuberculosis & ESI-Q-TOF & $\begin{array}{l}\text { Lipidomics with } \\
\text { transcriptomics for } \\
\text { regulatory network during } \\
\text { hypoxia }\end{array}$ \\
\hline & {$[48]$} & $\begin{array}{l}\text { M. tuberculosis } \\
\text { (35 strains) }\end{array}$ & ESI-QqQ & $\begin{array}{l}\text { Lipidomics with } \\
\text { genomics to investigate } \\
\text { MA metabolism }\end{array}$ \\
\hline
\end{tabular}




\section{FIGURE LEGEND}

Figure 1. Structures of mycobacterial lipids reviewed here. (A) Representative structure of major alpha, methoxy and keto mycolic acids of M. tuberculosis. As discussed in the text, related species synthesise mycolic acids with varying chain lengths. (B) Structure of PIMs. Dotted lines show the position of attachment of each mannose unit. (C) Structure of TMMs and TDMs. The mycolate substituents are those shown in Figure 1A. (D) Structure of DATs, TATs and PATs along with the major mycolipenic and mycocerosic acids that are bound to the trehalose core. (E) Structure of PGLs. (F) Structure of MPI. (G) Structure of SL-1 and SL-2. (H) Structure of TAGs and mmDAGs along with the major meromycolic acids that are bound to position $\mathrm{R}_{3}$ of mmDAGs. (I) Structure of PDIMs showing the major phthiocerols and phthiodiolones that make up the core of this lipid class. Abbreviations: DAT, diacyl trehalose; FA, fatty acid; MA, mycolic acid; mmDAG, monomeromycolyl diacylglycerol; MPI, mannosyl $\beta$-1-phosphoisoprenoid; PAT, polyacyl trehalose; PDIM, phthiocerol dimycoceroserate; PGL, phenolic glycolipid; PIM, phosphatidyl-myo-inositol mannoside; TDM, trehalose dimycolate; TMM, trehalose monomycolate; SL, sulfolipid; TAG, triacylglycerol; TAT, triacyl trehalose.

Figure 2. Analysis of mycolic acids by ESI-MS. (A) Fragmentation pathway for two isobaric alpha mycolic acids with 78 carbons in total and either a C22 or C24 alpha chain. Upon ionisation, a negative ion of $1136.1669 \mathrm{Da}$ is formed by loss of a proton from the carboxylic acid. Isolation followed by activation of this ion by CID gives fragments of $395.3895 \mathrm{Da}$ and $367.3582 \mathrm{Da}$ characteristic of C24 and C22 alpha chains respectively. (B) Negative mode mass spectrum recorded on an LTQ-Orbitrap showing mycolic acids extracted from M. tuberculosis strain N0145 [48]. The spectrum shows a series of alpha and methoxy mycolic acids. Each peak may be comprised of multiple isobaric species with varying alpha chain lengths. (C) Negative mode MS/MS spectrum recorded on an LTQ-Orbitrap after isolation of the ion at 1136.17 and activation by CID. The spectrum shows that the peak at 1136.17 is comprised of a mixture of mycolic acids with a C22 or C24 alpha chain. Abbreviations: CID, collision-induced dissociation; ESI, electrospray ionisation; $\mathrm{m} / \mathrm{z}$, mass to charge ratio; MS, mass spectrometry. 
A

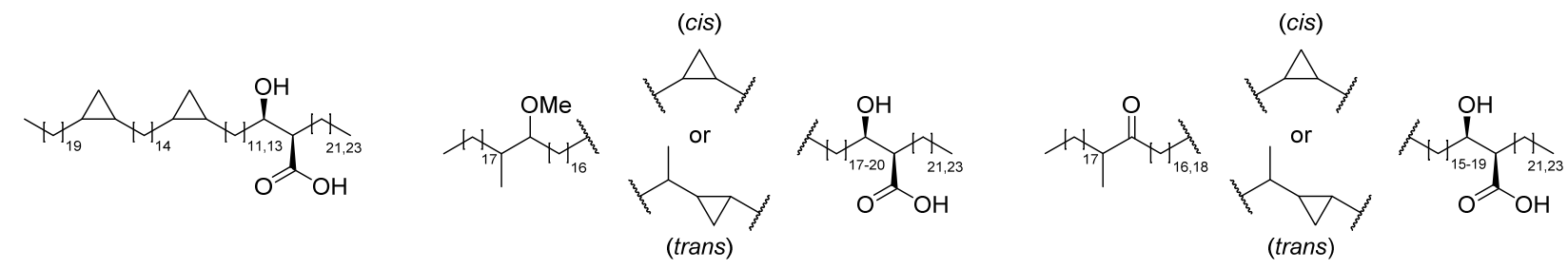

alpha MA

methoxy MA

keto MA

B

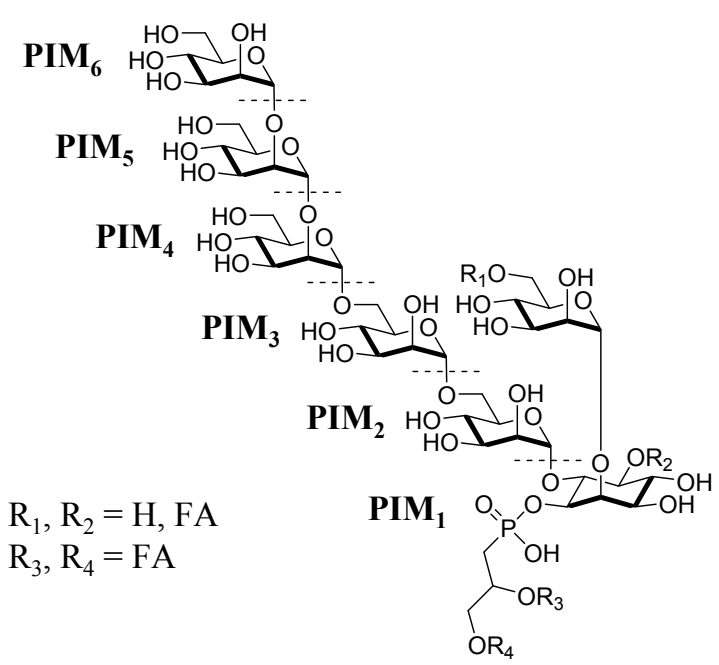

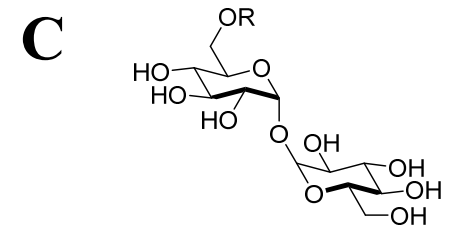

TMM

$(\mathrm{R}=$ mycolate $)$

D

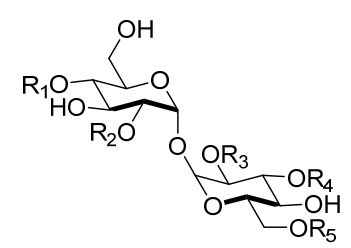

DAT $\left(R_{1}, R_{2}, R_{5}=H, R_{3}, R_{4}=X, Y, F A\right)$

TAT $\left(R_{1}, R_{2}=H, R_{3}, R_{4}, R_{5}=X, Y, F A\right)$

$\operatorname{PAT}\left(\mathrm{R}_{1}, \mathrm{R}_{2}, \mathrm{R}_{3}, \mathrm{R}_{4}, \mathrm{R}_{5}=\mathrm{X}, \mathrm{Y}, \mathrm{FA}\right)$

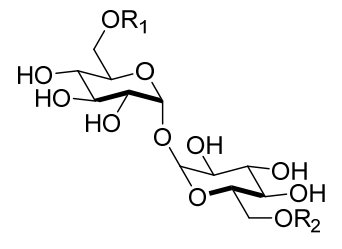

TDM

$\left(\mathrm{R}_{1}, \mathrm{R}_{2}=\right.$ mycolate $)$

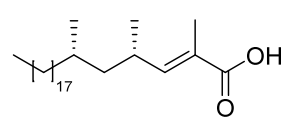

$\mathbf{X}$ (mycolipenic acid)

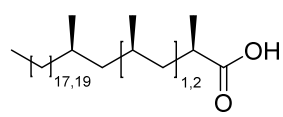

Y (mycocerosic acid)

$\mathbf{E}$

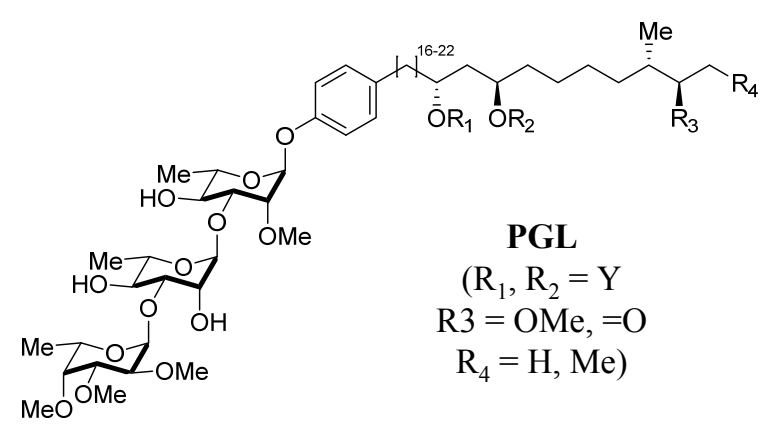

F

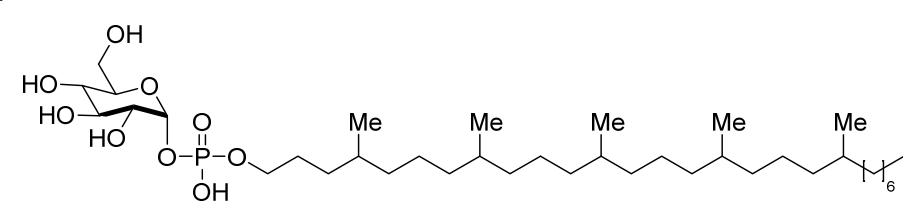

MPI

H

$$
\mathrm{R}_{1} \mathrm{O} \overbrace{\mathrm{OR}_{2}}^{\mathrm{OR}_{3}}
$$

TAG $\left(\mathrm{R}_{1}, \mathrm{R}_{2}, \mathrm{R}_{3}=\mathrm{FA}\right)$ $\operatorname{mmDAG}\left(\mathrm{R}_{1}, \mathrm{R}_{2}=\mathrm{FA}, \mathrm{R}_{3}=\mathrm{Z}\right)$

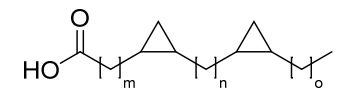

$\mathbf{Z}$ (meromycolic acid) $(\mathrm{m}+\mathrm{n}+\mathrm{o}=29-49)$

G

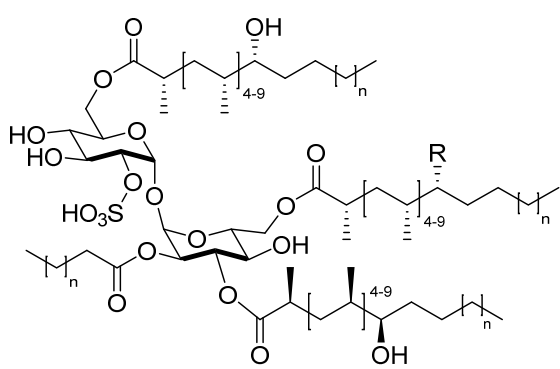

SL-1 $(\mathrm{R}=\mathrm{H})$

SL-2 $(\mathrm{R}=\mathrm{OH})$ phthiocerol A

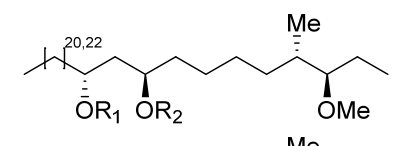

phthiocerol B

phthiodiolone

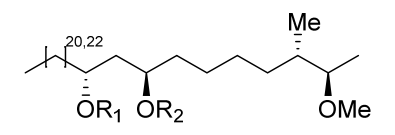

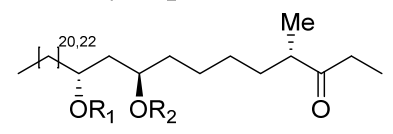

PDIM

$\left(\mathrm{R}_{1}, \mathrm{R}_{2}=\mathrm{Y}\right)$ 
A

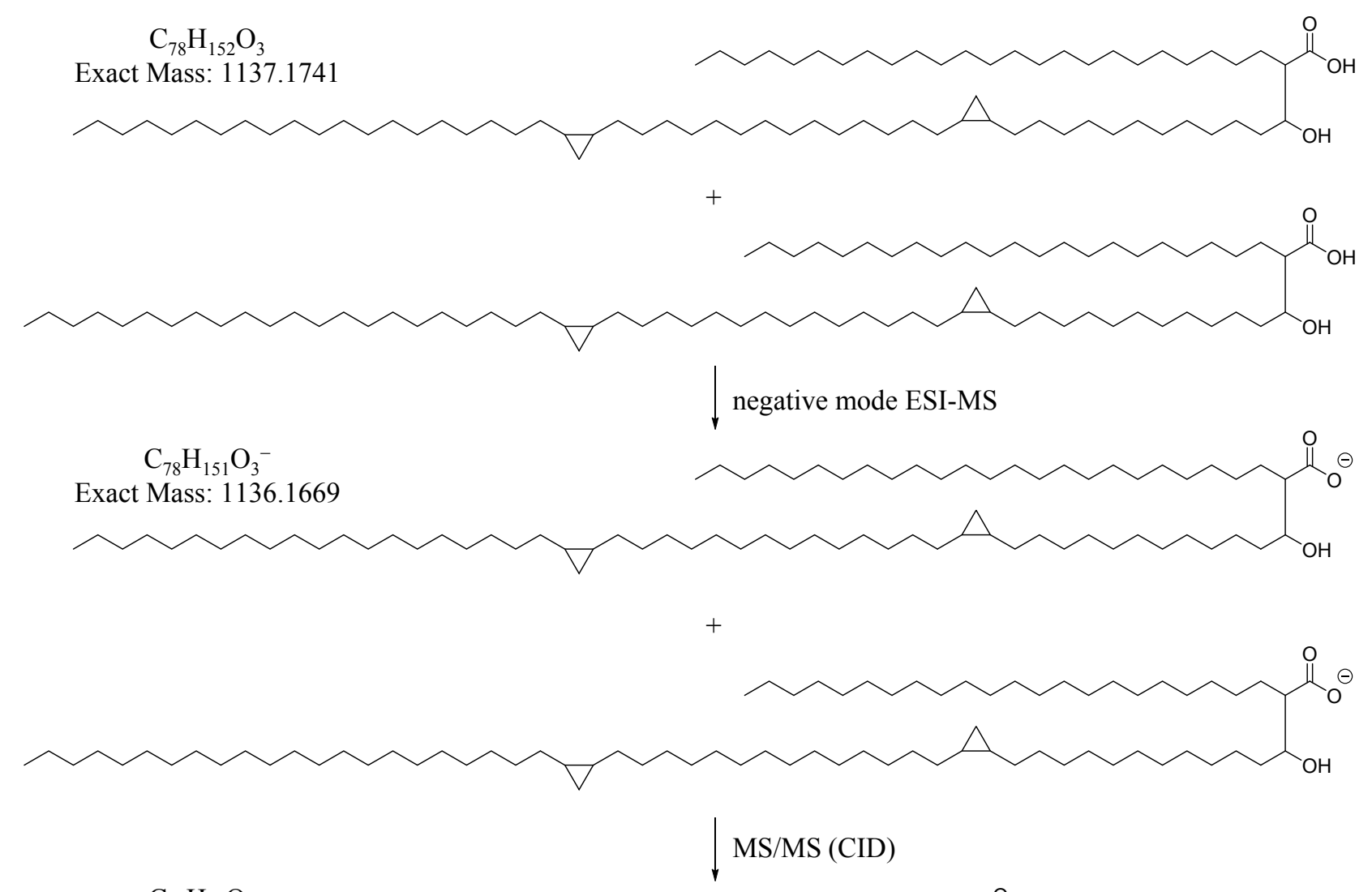

$\mathrm{C}_{26} \mathrm{H}_{51} \mathrm{O}_{2}^{-}$

Exact Mass: 395.3895

C24 alpha-chain fragment

$\mathrm{C}_{24} \mathrm{H}_{47} \mathrm{O}_{2}^{-}$

Exact Mass: 367.3582

B

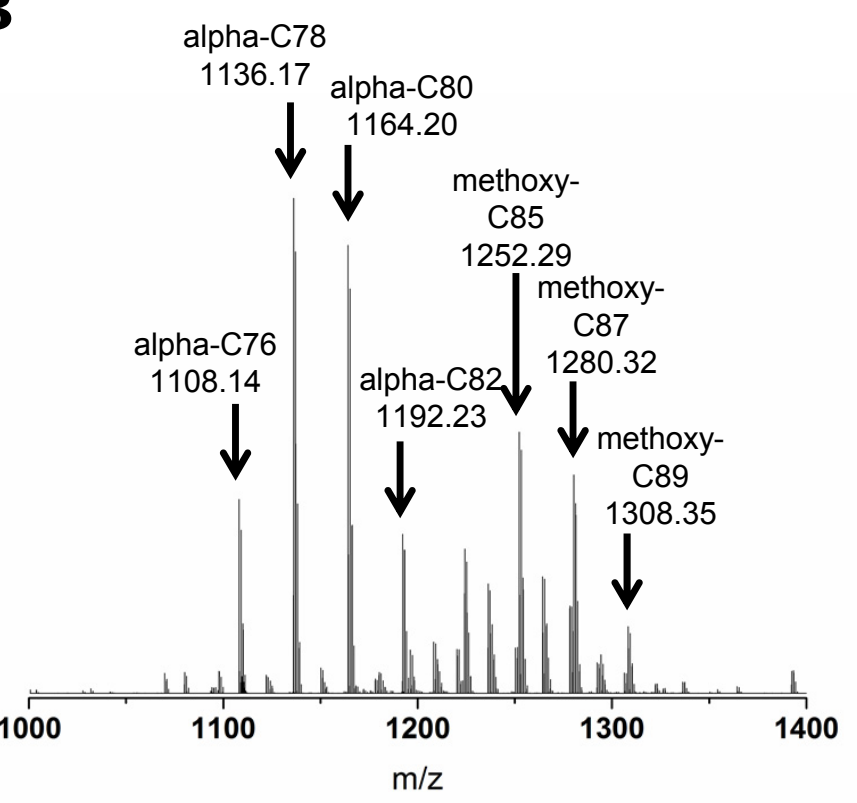

C C24 alpha

chain

395.39
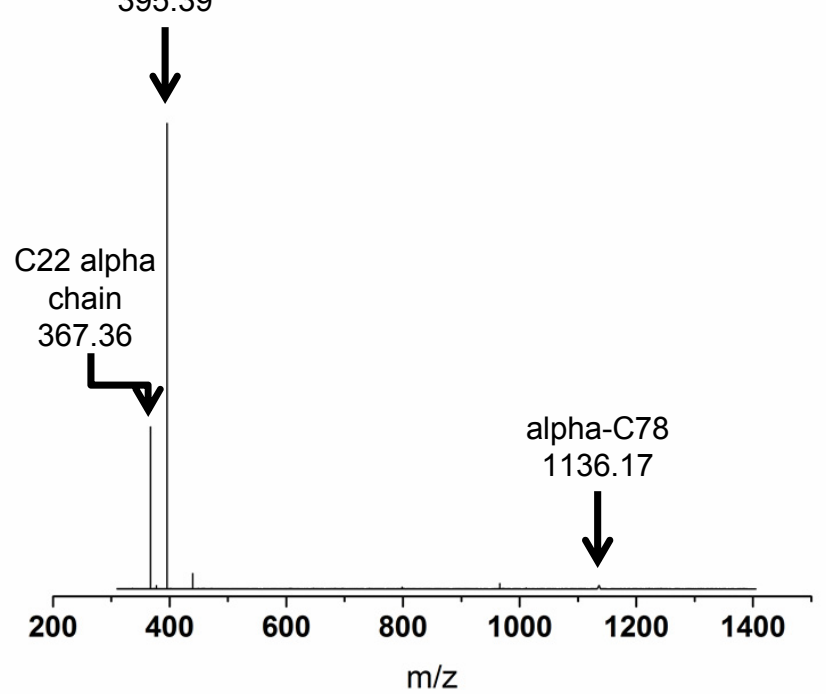